\title{
Six-Week Actual Use Study to Evaluate the Impact of Oral Tobacco-Derived Nicotine Pouches on Cigarette Smoking and Smokeless Tobacco Product Use Behaviors
}

\section{Stacey McCaffrey}

Altria Corporate Services Inc: Altria Client Services Inc

Jennifer Lewis

Altria Client Services Inc

Elizabeth Becker

Altria Client Services Inc

Andrea Vansickel

Altria Client Services Inc

Elsa Larson

Altria Client Services Inc

Mohamadi Sarkar ( $\square$ mohamadi.sarkar@altria.com )

Altria Client Services Inc https://orcid.org/0000-0002-1167-6140

\section{Research}

Keywords: oral nicotine, tobacco-derived nicotine, nicotine pouches, potentially reduced harm tobacco products

Posted Date: October 5th, 2021

DOl: https://doi.org/10.21203/rs.3.rs-951433/v1

License: (c) (1) This work is licensed under a Creative Commons Attribution 4.0 International License. Read Full License 


\section{Abstract}

\section{Background}

Oral tobacco-derived nicotine (OTDN) products are a rapidly emerging, innovative category of noncombustible tobacco products. These products are tobacco-leaf free and do not contain or generate many of the harmful and potentially harmful constituents (HPHCs) found in combustible cigarettes or smokeless tobacco (ST) products. OTDN products may therefore offer adult tobacco consumers a potentially reduced harm alternative. However, little is known about OTDN product use patterns or their ability to substitute for more harmful tobacco products.

Methods

We conducted an open-label, 2-phase, actual use study designed to characterize OTDN pouch product (on!® nicotine pouches - NP) use patterns, including impact on other tobacco use behaviors among adult tobacco consumers interested in using NP. Adult current cigarette smokers (AS) and/or adult ST users (ASTU) ( $n=1,147$ complete) who were not planning to quit were offered free choice of a portfolio of NP (seven flavors at five nicotine levels) to use at-home, ad-libitum, for 6-weeks following an initial 5-day trial. Participants responded to daily electronic surveys that captured the amount, frequency, and topography (e.g., time in mouth, placement in mouth) of NP and other tobacco product use.

Results

The majority (99 to 100\%) of study participants used NP throughout the 6-week period. Participants used 5-6 pouches/day of a variety of flavors and nicotine levels. At the end of 6 weeks, a modest proportion of AS (27\%) and a substantial proportion of ASTU (71\%) reported no use of cigarettes or ST respectively, while reporting continued use of NPs. Additionally, $39 \%$ of AS and $14 \%$ of ASTU reduced daily consumption of cigarettes or ST products, respectively by $50-99 \%$.

\section{Conclusions}

Participants found NPs acceptable with a sizeable proportion completely switching away from or substantially reducing (50-99\%) their cigarette or ST consumption by week 6 . Availability of a variety of flavors and nicotine levels appeared to facilitate the conversion to NP. These data suggest that, under the conditions of the study, NP can be a potential substitute for cigarettes or ST products and may offer harm reduction potential for adult smokers and smokeless tobacco users not interested in quitting.

\section{Trial Registration}

Note: This study is an observational study with tobacco products currently available in the marketplace for adult tobacco consumers. Since there is no intervention per se and we did not collect any biosamples this is not a "clinical study" and there is no appropriate registry for such behavioral observational studies, we do not believe that this requirement is applicable. 


\section{Background}

There is growing public health consensus that a continuum of risk exists within the range of tobacco products. Combustible tobacco products, such as conventional cigarettes, pose the greatest health risks, and noncombustible tobacco products, such as smokeless tobacco (ST) products, present relatively lower risks $(1,2)$. The evidence that stopping cigarette smoking leads to significant reductions in tobaccorelated morbidity and mortality is compelling and irrefutable (3). While complete tobacco cessation presents the best option for reducing morbidity and mortality, complete switching to noncombustible tobacco products may benefit those cigarette smokers who are unwilling or unable to quit all nicotine (4).

For noncombustible tobacco products to reach their true harm reduction potential, adult tobacco consumers (ATCs) must fully switch away from more harmful tobacco products. For example, epidemiological evidence clearly demonstrates that ST products, including snus, are far less risky than cigarettes $(4,5)$. Snus products have received considerable attention for their harm reduction potential in Sweden (6-8) and some in the U.S. have been authorized to be marketed as modified risk tobacco products (9).

Oral tobacco-derived nicotine (OTDN) products are a rapidly emerging, innovative category of noncombustible tobacco products that have not been well-investigated. The on! ${ }^{\circledR}$ nicotine pouches (NP, hereafter referred to as Test Products) assessed in the current study are OTDN products that are tobaccoleaf free and are thus devoid of, or contain substantially lower levels of, HPHCs relative to cigarettes and traditional, noncombustible tobacco products, such as snus and moist ST (10). The Test Products are available in a variety of flavors and nicotine levels (seven flavor varieties: wintergreen, mint, cinnamon, coffee, citrus, berry, and original; five nicotine levels per flavor: $1.5 \mathrm{mg}, 2 \mathrm{mg}, 3.5 \mathrm{mg}, 4 \mathrm{mg}$, and $8 \mathrm{mg}$ ) that provide choices for ATCs. The availability of multiple flavors and nicotine levels may facilitate the transition away from cigarettes, thus offering a potentially reduced harm alternative to adult smokers unable or unwilling to quit (11-15). However, to date there are no available published reports on the impact of using the Test Products on the continued use of or switching behavior from more harmful forms of tobacco.

The aim of this 6-week actual use study was to characterize tobacco use patterns and behaviors among ATCs of cigarettes and ST when provided the Test Products for use under near real-world conditions. The study sought to determine the frequency, amount, and patterns of Test Product use over time. This study also assessed the impact of Test Product use on cigarette and ST consumption patterns (e.g., reductions/increases in use), and complete switching behavior. Results of this study are intended to provide insights into the harm reduction potential of the Test Products, through investigation of use patterns, including complete switching behavior, among adult smokers and ST users.

\section{Methods}

\section{Study Design}


This multi-site, observational study was conducted by MarketView Research Group, Inc. (Edgewater, NJ), who fielded the study from August 16th to November 27th, 2019.

\section{Participants}

Adults of legal age to purchase tobacco products at the time this research was conducted (age 18 or older, depending on where the participant resided), up to age 65, were recruited from 81 research facilities around the United States by phone via third-party vendors' existing databases. Participants were selfreported current adult cigarette smokers (AS; reported smoking cigarettes "every day" or "some days" in the past 30 days), adult ST users (ASTU; reported using ST "everyday" or "some days" in the past 30days), or adult dual users of cigarettes and ST (ADU; reported cigarette and ST use "everyday" or "some days" in the past 30-days) at screening. All participants met lifetime criterion thresholds for tobacco use (i.e., $\geq 100$ cigarettes, >20 ST uses), were not planning to quit smoking cigarettes and/or using ST in the next 30 days and may have reported using other tobacco products (e.g., e-cigarettes, hookah, cigars). All participants self-reported that they were generally in good health (did not self-report allergies to menthol or phenylalanine, periodontal disease, gum disease or bleeding gums, open mouth sores or ulcers, cardiovascular disease, diabetes, high blood pressure, or cancer). Participants were excluded if they had ever used the Test Products, were unable to read, speak, or understand English, were employed by or in litigation with or had a close family member employed by or in litigation with a tobacco company, were pregnant or nursing, or participated in tobacco research lasting longer than 2-weeks in the past 12 months. All potential participants digitally signed an informed consent (IC) and protected health information (PHI) form. This study protocol and all related documents were approved by the Advarra Institutional Review Board (IRB), 6940 Columbia Gateway Drive, Suite 110, Columbia, MD 21046.

\section{Procedure}

Participants completed a brief telephone screening followed by an on-site consent and screening session. Once deemed eligible at screening and having provided informed consent, participants were shown a description of the Test Products and promotional materials (e.g., product line up, website images, and print materials) and asked about their intentions to try the Test Products using a validated 3-item Intention to Try scale (16). Participants open to trying the Test Products (i.e., their Intention to Try composite score corresponded to "somewhat agree," "agree," or "strongly agree") were invited to participate in a 5-day trial. On each of the five days, participants were instructed to use one of five flavor varieties[1] in at least one but up to three nicotine levels (2 mg, $4 \mathrm{mg}$, and $8 \mathrm{mg})$. Additionally, participants completed a daily written diary to determine the amount and duration of Test Product use as well as use of other tobacco products, if any.

At the end of the 5-day trial, participants returned to the study site and completed a brief survey to assess their intentions to use the Test Products using a validated 4-item Intention to Use scale (16). Participants who tried all five flavors in at least one nicotine level and who had intention to use the Test Products (i.e., their Intention to Use composite score corresponded to "somewhat agree," "agree," or "strongly agree") 
were invited to participate in the 6-week extended use period (second phase of the study). Participants signed another IC and $\mathrm{PHI}$ form pertaining to this second phase.

During the 6-week extended use period, participants had free choice of Test Products from the full product portfolio (any combinations of the seven flavor varieties with five nicotine levels per flavor), up to ten cans (20 pouches per can) per week. Participants took Test Products home to use ad libitum and received daily, automated e-mails with links to surveys containing questions related to the variety and amount of Test Product pouches used, the number of cigarettes smoked, and the number of ST use occasions, if any, during the previous day. Participants received additional questions pertaining to typical Test Product use occasions (time in mouth, where in mouth, and number of pouches used per occasion; "topography") once a week and recorded the amount and frequency of use of other tobacco products (excluding cigarettes and ST products) during the previous week. Participants returned to the study sites on a weekly basis, or as frequently as needed, to return unused Test Products and request new Test Products (of preferred flavor and nicotine level) for the coming week. At the end of week 6, participants responded to an online (e-mailed) end-of-study survey and returned all unused Test Products to the primary research organization.

\section{Data Analysis}

This study was descriptive in nature and data are reported on an unweighted basis. Results among the study groups and total study sample are described using proportions and frequency distributions or means, standard deviations, medians, and confidence intervals depending on the outcome measure. All upper/lower bounds were calculated at $95 \%$. All analyses were performed using Quantum software (Version 5.8), SPSS (Version 25), and R (Version 3.6). Only results from the 6-week period (phase 2) are reported here, as the 5-day trial (phase 1) was considered screening for inclusion. Data imputation was employed to account for missing data points[2] in weekly product use estimates, whereby the average product use for the non-missing days in a week was imputed into the missing day(s).

Prevalence of Test Product use, overall and by flavor and by nicotine level, was examined, as well as the frequency and amount of Test Product use, and the topography of Test Product use. Cigarette and ST use at the end of the study was also examined among participants using the Test Products. Each adult smoker participant (AS and ADU groups) who used the Test Products during Week 6 (Days 36-42) was classified into one of the following categories:

- No Cigarette Use (Switchers) - Did not use any cigarettes in Week 6

- Dual Use (50-99\% Reduction) - Reduced cigarettes per day (CPD) by 50-99\% in Week 6 compared to what was reported at screening

- Dual Use (1-49\% Reduction) - Reduced CPD by $1-49 \%$ in Week 6 compared to what was reported at screening

- Dual Use (No Change) - No change in CPD in Week 6 compared to what was reported at screening

- Dual Use (Increase) - Increase in CPD in Week 6 compared to what was reported at screening 
Adult ST user participants (ADU and ASTU groups) were classified into similar categories based on their ST use during Week 6 (e.g., No ST Use (Switchers), Dual Use (50-99\% Reduction), etc.).

\section{Results}

\section{Participants}

Of the 22,490 individuals who completed a brief initial telephone prescreening, the 2,700 that were considered eligible through the prescreening were invited to a research site to complete the full computerized screening. In total, 1,919 individuals completed the full screening and were enrolled in the 5 -day trial (phase 1). Eighty-seven percent of those participants $(n=1,590)$ were deemed eligible after phase 1 and enrolled in the 6-week period (phase 2). Seventy-two percent of the participants who enrolled in phase 2 completed the 6-week period and the End of Study Survey ("Final Study Sample" $n=1,147$ ); $n=$ 399 AS, $n=395$ ADU, and $n=353$ ASTU.

Demographic and tobacco product use information for the Final Study Sample is presented in Table 1.

\section{Prevalence of Test Product Use}

Almost all of the participants (99-100\%) reported using the Test Products on at least one day each week during the 6-week period. Each of the seven flavors was used by relatively high percentages of participants (i.e., 17\%-67\%) each week during the 6-week period (see Additional File 1 for the percentage of AS, ADU, and ASTU who used each flavor of the Test Products each week). AS, ADU, and ASTU reported using an average of $2.87(S D=1.33), 3.07(S D=1.44)$, and $2.97(S D=1.45)$ flavors during Week 1 respectively. By Week 6 these means declined to $2.32(S D=1.27), 2.30(S D=1.36)$, and $2.28(S D=1.34)$, respectively. Figure 1 depicts the percentage of participants who used each flavor at least once during the 6-week period.

The prevalence of nicotine level use by week is presented in Additional File 2. Across the three study groups, the $4 \mathrm{mg}$ nicotine pouches were used by the highest percentage of participants and the $1.5 \mathrm{mg}$ nicotine pouches were used by the lowest percentage of participants each week (Additional File 2) and overall (Figure 2) during the 6-week period.

Frequency and Amount of Test Product Use

Table 2 presents descriptive statistics pertaining to the frequency and amount of Test Product use among those participants who reported using the Test Products at least one day in a given week during the 6week period. AS, ADU, and ASTU reported using the Test Products seven days per week throughout the 6week period (Table 2). Average number of pouches per week remained relatively consistent over time (AS $=47.8-51.1 ; \mathrm{ADU}=51.4-53.5 ; \mathrm{ASTU}=53.5-58.2$.

The average number of days per week each of the different varieties of Test Products was used by participants was relatively similar across flavors (see Additional File 3 ) and across nicotine levels (see 
Additional File 4). Descriptive statistics for the number of Test Products used per week by flavor are presented in Additional File 5. Descriptive statistics for the number of Test Products used per week by nicotine level are presented in Additional File 6.

\section{Topography}

Overall, participants reported keeping the Test Products in their mouths approximately 12-13 minutes during a typical use occasion ( $A S=11.5, A D U=13.0, A S T U=13.1)$. These means appeared to rise slightly and gradually during the 6-week period; i.e., the average values between Day 1 and Day 42 changed by about 0.5 minutes, 0.7 minutes, and 1.0 minutes among AS, ADU, and ASTU, respectively.

Approximately $81 \%, 74 \%$, and $70 \%$ of AS, ADU, and ASTU, respectively, reported typically using one Test Product pouch per use occasion. These percentages remained relatively stable throughout the 6-week period.

Over $95 \%$ of AS, ADU, and ASTU reported that their typical placement of the Test Product pouches was either "under the top lip against the gum" or "under the bottom lip against the gum" each week. Typical pouch placement did not appear to change substantially over the 6-week period. Other product placements reported by a small number of participants included both the upper and lower lip, under the tongue, and between the gum and cheek.

Cigarette and ST Use at End of Study Among Participants Using the Test Products

In general, regardless of study group, the percentage of participants that reported using cigarettes, ST, and other tobacco products on at least one day during a given week trended downwards across the 6week period (as shown in the figures presented in Additional File 7).

\section{Change in Cigarette Smoking}

At Week $6,27 \%$ of AS switched completely to the Test Products (i.e., they reported using the Test Products during Week 6 and did not smoke any cigarettes), and 39\% reported dual using cigarettes and the Test Products while reducing their CPD by $50 \%$ or more from that reported at screening (Table 3 ). An additional $24 \%$ of AS reduced CPD by $1-49 \%$ at week 6 from that reported at screening. A relatively small proportion (3\%) of AS continued to smoke the same CPD as reported at baseline and also used Test Products. Additionally, $6 \%$ of the AS increased their CPD and also used Test Products. ADU exhibited similar use behavior with respect to change in CPD (Table 3).

Of note, the study participants in the "50-99\% Reduction in Week 6" group smoked more CPD at screening (15.3) than those in the "No Cigarettes in Week 6" group (9.3).

\section{Change in ST Use}

Among ADU using the Test Products during Week 6,78\% of participants switched to the Test Products from ST, and $9 \%$ reduced their ST use occasions by $50 \%$ or more from what was reported at screening. 
Five percent (5\%) of ADU used the Test Products while maintaining the same number of ST use occasions and $3 \%$ increased their ST use occasions from what they reported at screening. Similar findings were observed among ASTU (Table 3).

\section{Discussion}

Results of this study provide preliminary evidence of the harm reduction potential of the Test Products, particularly given that the study participants were not interested in quitting smoking and/or ST use. The majority of AS, ADU, and ASTU either switched completely or drastically reduced (50-99\%) their cigarette and ST consumption by week 6 . These results were observed in the absence of participants being instructed to switch to the Test Products. The vast majority of the study participants appeared to find the Test products acceptable. More than $90 \%$ reported using the Test Products daily and used five to six pouches per day. All seven flavors and five nicotine levels were reportedly used over the 6-week period. These observations in conjunction with the demonstrably lower levels of HPHCs (10) in the Test Products compared to cigarettes, suggest that the Test Products present a harm reduction opportunity among adult smokers and smokeless tobacco users .

The availability of flavor and nicotine level options and participants' free choice of product likely contributed to the switching rates and cigarette and ST reductions observed in this study. Over the 6-week ad libitum use period, all seven flavors of the Test Products were used by AS, ADU, and ASTU. As participants developed preferences, we observed a decrease in the average number of flavors and nicotine levels used each week over the 6-week period. This research study has also demonstrated that using a higher number of Test Product flavors was associated with fewer CPD during the 6-week period, independent of nicotine level (11). This finding suggests that the availability of flavor options may facilitate switching.

The Test Product use behaviors observed in the present study are in contrast with reports on other pouched tobacco products. For example, low rates of switching from cigarettes to snus (a pouched product containing tobacco leaf) has been reported in several studies (17-20). Unlike the snus products assessed in these studies, the Test Products in this study do not contain tobacco leaf and were offered in a variety of flavors and nicotine levels, which may have facilitated adoption and switching behavior. Additionally, in contrast to other studies with snus (17-20), this study enrolled participants who were open to trying and interested in continuing to use the Test Products. The differences between study designs and products likely account for the variation of findings across studies.

High rates of adoption of the Test Products in conjunction with the observed downward trends in the percentage of participants who used cigarettes, smokeless tobacco, and other tobacco products across study groups suggests that participants replaced these tobacco products with the Test Products. Furthermore, by Week 6, the majority of AS, ADU, and ASTU had either switched completely to the Test Products or reduced their cigarette/ST use by half or more (50-99\% reduction) from that reported at screening. Specifically, despite AS not planning to quit cigarettes at screening, $66 \%$ stopped smoking 
cigarettes and switched to the Test Products or substantially reduced their CPD by $50-99 \%$ from screening. Even higher rates of switching and reduction in ST use were observed among ADU and ASTU.

Moreover, as described elsewhere (21), the trajectory of reduction in cigarette consumption among AS who reduced cigarette consumption by $50-99 \%$ is similar to that observed for those who switched completely. These results suggest that, given sufficient time, some adult smokers who use the Test Products may eventually stop using cigarettes altogether. For those unable or unwilling to quit all tobacco complete switching represents the most desirable outcome however, adult smokers may need time before they change their tobacco use behavior (22), and dual use might be a transition phase before successfully switching to the Test Products. There is a significant body of evidence suggesting that reduction in smoking can be a potential pathway to smoking cessation (23). Undeniably cessation is the optimal outcome to achieve the proven reductions in smoking-related disease risks.

The results of this study should be considered in the context of its strengths and limitations. For example, the design of this actual use study strikes a balance between internal and external validity. The two-phase, ambulatory assessment of self-reported tobacco product use patterns among AS, ADU, and ASTU adds to the robustness of the study design. In order to participate in the full study, participants must have expressed intention to try (5-day trial) and intention to use (6-week period) the products. This requirement increased the likelihood that the tobacco use patterns observed in the current study would more closely resemble those of adult tobacco consumers who are likely to try and/or to use the products under real-world conditions. Participants had the choice to continue using cigarettes, smokeless tobacco, and other tobacco products ad libitum in their natural environments during the study. They were also given free choice of Test Products to use ad libitum. These aspects of the study design strengthened the robustness of our findings. That is, if participants had been randomized to use just one flavor or if their use of other tobacco products had been restricted, this would have limited external validity. Another strength of the study was the daily collection of self-reported tobacco use data during the 6-week period. This likely decreased recall bias and improved accuracy of data.

However, there were also some limitations of this study, which should be taken into consideration when interpreting the results. First, although we recruited the participants based on soft quota targets for key demographics for each study group based on the 2018 National Health Interview Survey, results from the current study may not be generalizable to the population for the current time period. Furthermore, the study participants were not planning to quit their tobacco products, further limiting the generalizability to all tobacco users. Second, participants received Test Products at no cost during the study, which may have led them to use the products differently than if they had paid for them. For example, it might be hypothesized that some participants switched to the Test Products because the product was free. Although it is not possible to determine the extent to which a free product effect may account for some of the observed findings, the differential rates of switching behavior observed across study groups suggests that a free product effect does not fully account for product use behavior. Finally, we relied on selfreported tobacco use and did not attempt to biochemically verify the change in cigarette consumption. However, previous research demonstrates a strong relationship between self-reported tobacco product 
use among US adults and serum cotinine levels (24), providing support for the validity of this selfreported data.

\section{Conclusion}

In the current study, we observed that most participants adopted the Test Products and many completely switched away from or drastically reduced (50-99\%) their cigarette or ST consumption by week 6 . These data suggest that, at least in this study population and under the conditions of the study, the Test Products can serve as a substitute for cigarettes or ST products. Availability of a variety of flavors and nicotine levels may facilitate conversion to these potentially reduced harm products. In conjunction with research demonstrating a reduction in HPHCs (10), the observed product use behavior from this study provides support for the harm reduction potential of the Test Products.

\section{Abbreviations}

AS = adult cigarette smokers

$\mathrm{ADU}=$ adult dual users

ASTU = adult smokeless tobacco product users

ATCs = adult tobacco consumers

$\mathrm{Cl}=$ confidence interval

$\mathrm{CPD}=$ cigarettes per day

$\mathrm{HPHC}=$ harmful and potentially harmful constituents

$\mathrm{IC}=$ informed consent

IRB = institutional review board

$\mathrm{NA}=$ not applicable

$\mathrm{NP}=$ on! $@$ nicotine pouches

OTDN = oral tobacco-derived nicotine

$\mathrm{PHI}=$ protected health information

$\mathrm{SD}=$ standard deviation

$\mathrm{ST}=$ smokeless tobacco 


\section{Declarations}

\section{Ethics approval and consent to participate}

This research was approved by the Advarra Institutional Review Board (reference number MOD00477846) and the participants received complete information about the study before agreeing with an informed consent statement.

\section{Consent for publication}

Not applicable.

\section{Availability of data and materials}

Reasonable requests for datasets and/or analyses presented in this manuscript will be considered as appropriate, recognizing that the data is currently subject to review as part of a proprietary product application pending with FDA.

\section{Competing interests}

At the time this research was conducted, all authors were employees of Altria Client Services LCC.

\section{Funding}

This work was funded by Altria Client Services LLC.

\section{Authors' contributions}

Both H.E.L. and S.A.M were principal investigators of the study. S.A.M. drafted the manuscript, and all authors were involved in writing and/or editing the manuscript. All authors read and approved the final manuscript.

\section{Acknowledgements}

The authors are grateful to Meghan L Thompson, PharmD, PhD for supporting the writing and coordinating the review of the manuscript.

\section{References}


1. Cappelleri JC, Bushmakin AG, Baker CL, Merikle E, Olufade AO, Gilbert DG. Confirmatory factor analyses and reliability of the modified cigarette evaluation questionnaire. Addict Behav. 2007;32(5):912-23.

2. Gottlieb S, Zeller M. A Nicotine-Focused Framework for Public Health. N Engl J Med. 2017;377(12):1111-4.

3. Smoking Cessation. A Report of the Surgeon General, (2020).

4. Zeller M, Hatsukami D, Strategic Dialogue on Tobacco Harm Reduction G. The Strategic Dialogue on Tobacco Harm Reduction: a vision and blueprint for action in the US. Tob Control. 2009;18(4):324-32.

5. Fisher MT, Tan-Torres SM, Gaworski CL, Black RA, Sarkar MA. Smokeless tobacco mortality risks: an analysis of two contemporary nationally representative longitudinal mortality studies. Harm Reduct J. 2019;16(1):27.

6. Clarke E, Thompson K, Weaver S, Thompson J, O'Connell G. Snus: a compelling harm reduction alternative to cigarettes. Harm Reduction Journal. 2019;16(1):62.

7. Royal College of Physicians. Nicotine without smoke: Tobacco harm reduction. London: Royal College of Physicians,; 2016. Report No.: 978-1-86016-600-6.

8. Lee PN. Epidemiological evidence relating snus to health--an updated review based on recent publications. Harm reduction journal. 2013;10:36-.

9. $\quad$ FDA grants first-ever modified risk orders to eight smokeless tobacco products [press release]. October 222019.

10. Wagner K, Brown A, Jin C, Sharifi M, Lopez V, Ballentine R, et al. Characterization of on! ${ }^{\circledR}$ Nicotine Pouches - Part 1: HPHCs. 26th Society for Research on Nicotine and Tobacco; March 11-14, 2020; New Orleans, LA.

11. McCaffrey S, Lewis J, Cheng H, Larson H, Becker E, Sarkar M. Examination of Association Between Flavors and Nicotine Strengths of an Oral Tobacco-Derived Nicotine Pouch Product and Cigarettes per Day Over Six Weeks of Ad Libitum Use. 27th Annual Meeting of Society for Research on Nicotine \& Tobacco; Feb. 24-27, 2021.

12. Chen JC. Flavored E-cigarette Use and Cigarette Smoking Reduction and Cessation-A Large National Study among Young Adult Smokers. Subst Use Misuse. 2018;53(12):2017-31.

13. Russell C, Haseen F, McKeganey N. Factors associated with past 30-day abstinence from cigarette smoking in a non-probabilistic sample of 15,456 adult established current smokers in the United States who used JUUL vapor products for three months. Harm Reduct J. 2019;16(1):22.

14. Friedman AS, Xu S. Associations of Flavored e-Cigarette Uptake With Subsequent Smoking Initiation and Cessation. JAMA Netw Open. 2020;3(6):e203826.

15. Vansickel A, McCaffrey S. Flavors in potentially reduced harm products: their role in continued use and switching behaviors among adult tobacco consumers. 27th Annual Meeting of Society for Research on Nicotine \& Tobacco; Feb. 24-27, 2021.

16. McCaffrey S, Black R, Plunkett S. Psychometric Evaluation of Behavioral Intention Item Functioning Across Tobacco Product Categories. 26th Society for Research on Nicotine and Tobacco; March 11-14, 2020; New Orleans, LA.

\section{Tables}


Table 1. Participant demographics and tobacco use history reported at screening 


\begin{tabular}{|c|c|c|c|}
\hline & AS & ADU & ASTU \\
\hline & $n=399$ & $n=395$ & $n=353$ \\
\hline Gender, \% Male & 46.9 & 89.9 & 95.8 \\
\hline Age, Mean \pm SD (Median) & $\begin{array}{l}42.9 \pm 12.0 \\
(43.0)\end{array}$ & $\begin{array}{l}39.4 \pm 11.2 \\
(38.0)\end{array}$ & $\begin{array}{l}41.9 \pm 11.7 \\
(42.0)\end{array}$ \\
\hline Race/Ethnicity, \% White/Caucasian & 71.2 & 87.3 & 83.6 \\
\hline Median Income Range & $\begin{array}{l}\$ 40,000- \\
\$ 49,999\end{array}$ & $\begin{array}{l}\$ 50,000- \\
\$ 59,999\end{array}$ & $\begin{array}{l}\$ 50,000- \\
\$ 59,999\end{array}$ \\
\hline \multicolumn{4}{|l|}{ Education, \% } \\
\hline High school or less & 47.9 & 45.6 & 38.8 \\
\hline Some college or more & 52.1 & 54.4 & 61.2 \\
\hline \multicolumn{4}{|l|}{ Employment Status, \% } \\
\hline Employed & 79.7 & 86.8 & 89.5 \\
\hline Not Employed & 19.6 & 12.9 & 10.2 \\
\hline Prefer not to answer & 0.8 & 0.3 & 0.3 \\
\hline \multicolumn{4}{|l|}{ Region, \% } \\
\hline Northeast & 18.1 & 17.2 & 10.8 \\
\hline South & 39.4 & 43.0 & 41.6 \\
\hline Midwest & 21.3 & 18.0 & 23.5 \\
\hline West & 21.3 & 21.8 & 24.1 \\
\hline \multicolumn{4}{|l|}{ Current past 30-day use, $\%$} \\
\hline Cigarettes & 100.0 & 100.0 & NA \\
\hline Any smokeless tobacco product & NA & 100.0 & 100.0 \\
\hline Chewing tobacco & NA & 41.0 & 33.7 \\
\hline Dip/snuff & NA & 70.4 & 70.3 \\
\hline Snus pouches & NA & 43.3 & 33.4 \\
\hline Cigars & 24.1 & 32.9 & 4.5 \\
\hline E-vapor products & 16.3 & 27.9 & 6.2 \\
\hline Pipe (regular pipe, hookah) & 6.8 & 16.0 & 0.6 \\
\hline Oral tobacco-derived nicotine products & 3.8 & 17.2 & 8.8 \\
\hline
\end{tabular}




\begin{tabular}{|llll|}
\hline $\begin{array}{l}\text { Past 7-day use } \\
\text { (Median) }\end{array}$ & $6.5 \pm 1.3(7.0)$ & $6.0 \pm 1.7(7.0)$ & NA \\
\hline Cigarettes per day, Mean \pm SD (Median) & $12.3 \pm 8.8(10.0)$ & $10.3 \pm 8.0(9.0)$ & NA \\
\hline $\begin{array}{l}\text { Number of days used ST, Mean } \pm \text { SD } \\
\text { (Median) }\end{array}$ & NA & $4.3 \pm 2.3(4.0)$ & $5.6 \pm 1.9(7.0)$ \\
\hline $\begin{array}{l}\text { Number of ST uses per day, Mean } \pm \text { SD } \\
\text { (Median) }\end{array}$ & NA & $6.0 \pm 6.93(4.0)$ & $6.9 \pm 7.2(5.0)$ \\
\hline
\end{tabular}

Table 1 legend: Summary of participant demographic characteristics and tobacco use history selfreported at screening for participants in the final study sample. NA=Not applicable; SD=standard deviation; AS=Adult Cigarette Smokers; ADU=Adult Dual Users; ASTU=Adult Smokeless Tobacco Product Users.

\section{Table 2. Descriptive statistics for frequency and amount of Test Product used per Week}

\section{Table 3. Changes in cigarette and smokeless tobacco consumption from screening to Week 6 among those participants using the Test Products at Week 6}

Table 3 legend: Based on cigarette/smokeless tobacco product consumption at Week 6, participants were classified into one of the 5 tobacco use groups (e.g., No ST Products in Week 6, 50-99\% Reduction in Week 6, etc.). This table presents the number and percentage of participants from each study group who were classified into each tobacco use group, as well as the mean CPD/ST use occasions reported at screening and Week 6. AS = Adult Cigarette Smokers; ADU = Adult Dual Users; ASTU = Adult Smokeless Tobacco Product Users; $\mathrm{Cl}$ = confidence interval; $\mathrm{CPD}=$ cigarettes per day; $\mathrm{ST}$ = smokeless tobacco.

\section{Figures}




\section{Week 1}

Week 2

$n=399$

$n=399$

$n=399$

$6.9 \pm 0.5$

$6.9 \pm 0.5$

$6.8 \pm 0.6$

$6.8 \pm 0.7$

$6.9 \pm 0.6$

$6.8 \pm 0.7$

Mean $\pm S D$

7.0

7.0

7.0

7.0

7.0

7.0

Median

Test Products

Used per Week

Mean $\pm S D$

Median

$51.1 \pm 52.9$

$49.0 \pm 37.4$

$47.8 \pm 34.5$

$49.1 \pm 39.6$

$48.5 \pm 35.5$

$48.7 \pm 35.9$

38.0

38.0

38.0

40.0

39.5

38.8

Test Products

Used per Daya

Mean $\pm S D$

$7.3 \pm 7.6$

$7.0 \pm 5.4$

$6.8 \pm 4.9$

$7.0 \pm 5.7$

$6.9 \pm 5.1$

$7.0 \pm 5.1$

Median

5.4

5.4

5.4

5.7

5.6

5.5

\section{ADU}

Number of:

$n=395$

$n=395$

$n=393$

$n=394$

$n=393$

$n=393$

Days Test

Product Used per

Week

$6.9 \pm 0.4$

$6.9 \pm 0.5$

$6.9 \pm 0.5$

$6.9 \pm 0.6$

$6.9 \pm 0.6$

$6.8 \pm 0.8$

Mean $\pm S D$

7.0

7.0

7.0

7.0

7.0

7.0

Median

Test Products

Used per Week

Mean $\pm S D$

Median

\section{$53.4 \pm 36.4 \quad 51.4 \pm 34.2$}

$52.2 \pm 36.9$

$53.5 \pm 39.1$

$53.0 \pm 38.4$

$53.4 \pm 39.1$

42.0

42.0

43.0

42.5

43.0

42.0

Test Products

Used per Daya

Mean $\pm S D$

$7.6 \pm 5.2$

$7.3 \pm 4.9$

$7.5 \pm 5.3$

$7.6 \pm 5.6$

$7.6 \pm 5.5$

$7.6 \pm 5.6$

6.0

6.0

6.1

6.1

6.1

6.0

Median

ASTU

Number of:

$n=353$

$n=353$

$n=353$

$n=353$

$n=352$

$n=348$ 
Days Test

Product Used per

Week

$6.9 \pm 0.5$

$6.9 \pm 0.4$

$6.9 \pm 0.6$

$6.9 \pm 0.5$

$6.8 \pm 0.7$

$6.9 \pm 0.5$

Mean $\pm S D$

7.0

7.0

7.0

7.0

7.0

7.0

Median

Test Products

Used per Week

Mean $\pm S D$

$58.2 \pm 44.7 \quad 54.4 \pm 41.5$

$53.5 \pm 39.7$

$54.1 \pm 41.2$

$53.5 \pm 40.9$

$54.2 \pm 38.9$

Median

45.0

43.0

42.0

42.0

42.0

43.0

Test Products

Used per Day ${ }^{\mathrm{a}}$

Mean $\pm S D$

$8.3 \pm 6.3$

$7.8 \pm 5.9$

$7.6 \pm 5.7$

$7.7 \pm 5.9$

$7.6 \pm 5.8$

$7.7 \pm 5.6$

Median
6.4
6.1
6.0
6.0
6.0

6.1

Table 2 legend: Descriptive statistics pertaining to the frequency and amount of Test Product use among those participants who reported using the Test Products at least one day in a given week

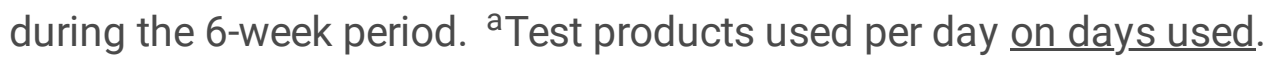

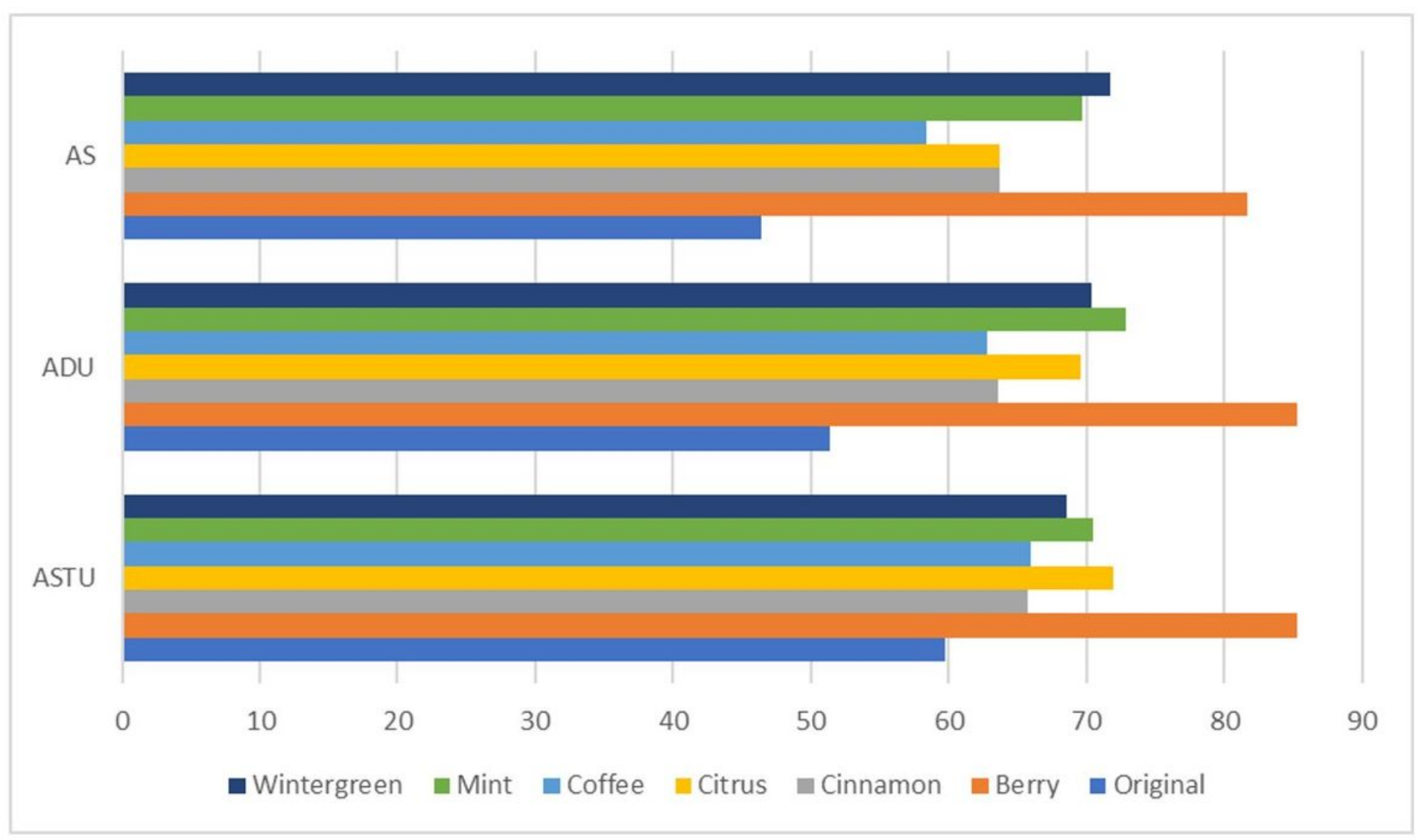

Figure 1 


\begin{tabular}{|c|c|c|c|c|}
\hline $\begin{array}{l}\text { Week } 6 \text { Tobacco Use } \\
\text { Category }\end{array}$ & $n$ & $\begin{array}{l}\% \text { of } \\
\text { Total }^{a} \\
(95 \% \mathrm{Cl})\end{array}$ & $\begin{array}{l}\text { Mean CPD/ST use } \\
\text { occasions per day } \\
\text { at screening }\end{array}$ & $\begin{array}{l}\text { Mean CPD/ST use } \\
\text { occasions per day at } \\
\text { Week } 6\end{array}$ \\
\hline \multicolumn{5}{|l|}{ AS } \\
\hline $\begin{array}{l}\text { Switchers (No Cigarettes } \\
\text { in Week 6) }\end{array}$ & 109 & $\begin{array}{l}27.32 \\
(22.95 \\
31.69)\end{array}$ & 9.25 & 0.00 \\
\hline $\begin{array}{l}\text { Dual Use: } 50-99 \% \\
\text { Reduction in Week } 6\end{array}$ & 156 & $\begin{array}{l}39.10 \\
(34.31 \\
43.89)\end{array}$ & 15.26 & 4.04 \\
\hline $\begin{array}{l}\text { Dual Use: 1-49\% } \\
\text { Reduction in Week } 6\end{array}$ & 94 & $\begin{array}{c}23.56 \\
(19.39 \\
27.72)\end{array}$ & 12.73 & 8.94 \\
\hline $\begin{array}{l}\text { Dual Use: No Change in } \\
\text { Week } 6\end{array}$ & 12 & $\begin{array}{l}3.01 \\
(1.33 \\
4.68)\end{array}$ & 7.67 & 7.67 \\
\hline $\begin{array}{l}\text { Dual Use: Increase in } \\
\text { Week } 6\end{array}$ & 25 & $\begin{array}{l}6.27 \\
(3.89 \\
8.64)\end{array}$ & 7.00 & 10.37 \\
\hline \multicolumn{5}{|c|}{ ADU (Cigarette Consumption) } \\
\hline $\begin{array}{l}\text { Switchers (No Cigarettes } \\
\text { in Week 6) }\end{array}$ & 113 & $\begin{array}{l}28.61 \\
(24.15 \\
33.06)\end{array}$ & 8.23 & 0.00 \\
\hline $\begin{array}{l}\text { Dual Use: } 50-99 \% \\
\text { Reduction in Week } 6\end{array}$ & 121 & $\begin{array}{c}30.63 \\
(26.09 \\
35.18)\end{array}$ & 14.06 & 3.93 \\
\hline $\begin{array}{l}\text { Dual Use: 1-49\% } \\
\text { Reduction in Week } 6\end{array}$ & 101 & $\begin{array}{l}25.57 \\
(21.27 \\
29.87)\end{array}$ & 10.75 & 7.52 \\
\hline $\begin{array}{l}\text { Dual Use: No Change in } \\
\text { Week } 6\end{array}$ & 15 & $\begin{array}{l}3.80 \\
(1.91 \\
5.68)\end{array}$ & 7.67 & 7.67 \\
\hline $\begin{array}{l}\text { Dual Use: Increase in } \\
\text { Week } 6\end{array}$ & 43 & $\begin{array}{c}10.89 \\
(7.81 \\
13.96)\end{array}$ & 5.26 & 8.14 \\
\hline \multicolumn{5}{|l|}{ ADU (ST Consumption) } \\
\hline $\begin{array}{l}\text { Switchers: No ST } \\
\text { Products in Week } 6\end{array}$ & 308 & $\begin{array}{l}77.97 \\
(73.89 \\
82.06)\end{array}$ & 6.33 & 0.00 \\
\hline $\begin{array}{l}\text { Dual Use: } 50-99 \% \\
\text { Reduction in Week } 6\end{array}$ & 34 & $\begin{array}{l}8.61 \\
(5.84 \\
11.37)\end{array}$ & 6.97 & 1.58 \\
\hline
\end{tabular}




\begin{tabular}{|c|c|c|c|c|}
\hline $\begin{array}{l}\text { Dual Use: } 1-49 \% \\
\text { Reduction in Week } 6\end{array}$ & 18 & $\begin{array}{l}4.56 \\
(2.50 \\
6.61)\end{array}$ & 6.50 & 4.70 \\
\hline $\begin{array}{l}\text { Dual Use: No Change in } \\
\text { Week } 6\end{array}$ & 21 & $\begin{array}{l}5.32 \\
(3.10 \\
7.53)\end{array}$ & 0.38 & 0.38 \\
\hline $\begin{array}{l}\text { Dual Use: Increase in } \\
\text { Week } 6\end{array}$ & 12 & $\begin{array}{l}3.04 \\
(1.35 \\
4.73)\end{array}$ & 2.83 & 4.68 \\
\hline \multicolumn{5}{|l|}{ ASTU } \\
\hline $\begin{array}{l}\text { Switchers: No ST } \\
\text { Products in Week } 6\end{array}$ & 250 & $\begin{array}{c}70.82 \\
(66.08 \\
75.56)\end{array}$ & 6.34 & 0.00 \\
\hline $\begin{array}{l}\text { Dual Use: } 50-99 \% \\
\text { Reduction in Week } 6\end{array}$ & 49 & $\begin{array}{c}13.88 \\
(10.27 \\
17.49)\end{array}$ & 11.84 & 2.61 \\
\hline $\begin{array}{l}\text { Dual Use: } 1-49 \% \\
\text { Reduction in Week } 6\end{array}$ & 21 & $\begin{array}{l}5.95 \\
(3.48, \\
8.42)\end{array}$ & 6.95 & 4.77 \\
\hline $\begin{array}{l}\text { Dual Use: No Change in } \\
\text { Week } 6\end{array}$ & 10 & $\begin{array}{l}2.83 \\
(1.10 \\
4.56)\end{array}$ & 2.00 & 2.00 \\
\hline $\begin{array}{l}\text { Dual Use: Increase in } \\
\text { Week } 6\end{array}$ & 18 & $\begin{array}{l}5.10 \\
(2.80, \\
7.39)\end{array}$ & 3.61 & 5.50 \\
\hline
\end{tabular}

Percentage of Participants Using Each Flavor during the 6-Week Period. Percentage of participants who used each flavor of the Test Products during the 6-week period. For example, $72 \%$ of AS used Wintergreen on at least one day during the 6-week period. 


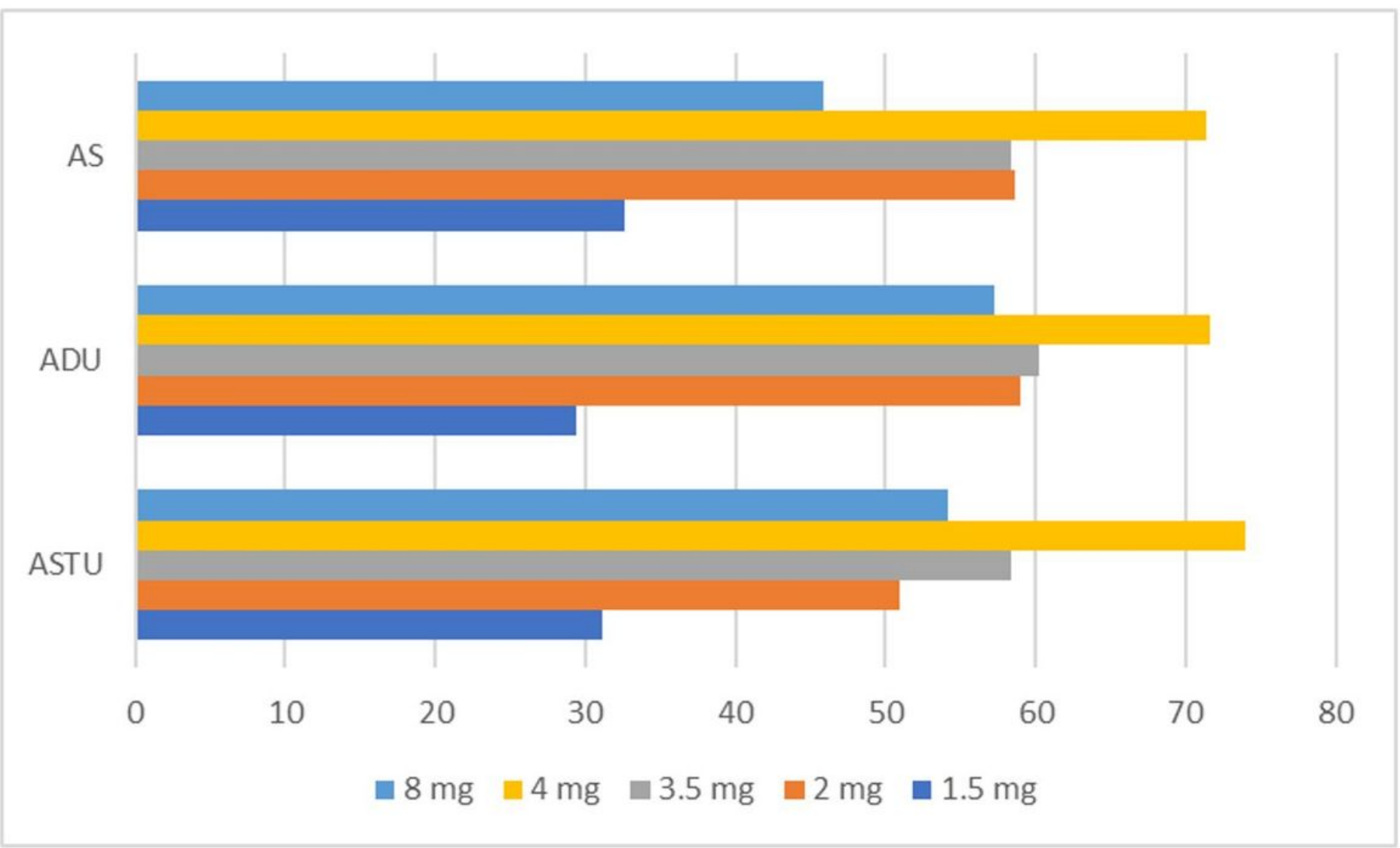

\section{Figure 2}

Percentage of Participants Using Each Nicotine Level during the 6-Week Period. Percentage of participants who used each nicotine level of the Test Products during the 6-week period. For example, $33 \%$ of AS used $1.5 \mathrm{mg}$ nicotine level of the Test Products at some time during the 6-week period. AS=Adult Cigarette Smokers; ADU=Adult Dual Users; ASTU=Adult Smokeless Tobacco Product Users.

\section{Supplementary Files}

This is a list of supplementary files associated with this preprint. Click to download.

- AdditionalFile7.docx

- AdditionalFile1.docx

- AdditionalFile2.docx

- AdditionalFile3.docx

- AdditionalFile4.docx

- AdditionalFile5.docx

- AdditionalFile6.docx 